Yuanbiao Qiao*, Wenhao Qin and Qingshan Li*

\title{
Sulfates of Sorghum vinegar residue waste as potential catalysts
}

https://doi.org/10.1515/gps-2017-0042

Received April 5, 2017; accepted June 5, 2017; previously published online September 5, 2017

Abstract: A sealed wet curing design on sulfating Sorghum vinegar residue waste is drilled with sulfo/sulfoalkyl chemicals. Sulfation degrees in the case of reactions with sulfuric acid, sodium sulfite, sulfamic acid, benzenesulfonic acid, $p$-toluenesulfonic acid and sodium laurilsulfate range from 14.7 to $36.9 \mathrm{mg}$ sulfo per gram waste. The C-O-S formation gives rise to accessional noncrystallinity and mesoporosity of morphology, a character for potential alternatives in catalysis. Waste sulfates are employed as candidates in catalyzing the condensation of $\alpha$-glycolic acid and urea substitutes for yielding imidazolidine2,4-dione derivatives. Catalytic and specific activities in terms of derivative productions are 2.6-34.3 $\left(\times 10^{3} \mathrm{U}\right)$ and $0.8-5.4 \mathrm{U} / \mathrm{mg}$ sulfo, together with the maximal yields of 49.4\%-97.6\%. Chemical linkage of sulfo/sulfoalkyl groups manifests synergism with intrinsic acidity of waste with respect to catalysis. A rational design of green chemistry is guaranteed by no excess of chemicals and easy recyclability of catalysts.

Keywords: agroindustrial waste reutilization; heterogeneous catalyst; imidazolidine-2,4-dione derivatives; Sorghum vinegar residue (SVR); sulfation modification.

\section{Introduction}

Sorghum vinegar residue (SVR) waste stacking is currently a severe burden to agroindustrial processing fields in China and other nations. SVR is an acidic by-product remainder obtained during the process of two-step fermentation from Sorghum with lactic acid bacteria and acetic acid bacteria [1]. A large quantity of approximately

*Corresponding authors: Yuanbiao Qiao, Graduate Institute of Pharmaceutical Chemistry, Luliang University, Shanxi 033001, P.R. China, e-mail: qyb_0222@sina.com; and

Qingshan Li, College of Traditional Chinese Pharmacology, Shanxi University of Traditional Chinese Medicine, Shanxi 030619, P.R. China; and School of Pharmaceutical Sciences, Shanxi Medical University, Shanxi 030001, P.R. China, e-mail: sxlqs2012@163.com Wenhao Qin: Department of Chemistry and Chemical Engineering, Luliang University, Shanxi 033001, P.R. China
1.8-2.2 million tons is generated per year as per China's annual reports [2-6]. Typical incineration, open discharge, and land filling of waste show strong pluripotency on water pollution, ecological toxicity, and regulatory information over a long period of duration. To diminish undesirable impacts of SVR, biological efforts for anaerobic digestion in getting energy-rich biogas [2-4], solidstate fermentation for producing bioorganic fertilizer [5, 7] and phytase [6], and bacterial biosynthesis of cellulose [8] have been performed. However, inapplicable carbon to nitrogen ratios of SVR and acidification of culture media are major limitations. Other attempts on preparing activated carbon particles and fibre matrix-based materials were tried by calcinating and impregnating waste [9]. All efforts devote to an actually little part of the list of reutilization of waste. A good design in much effective and ecofriendly fashion is interesting [4].

Sorghum vinegar residue is chemically composed of polymeric carbohydrate, protein, and other fractions [2, 4]. Polymeric carbohydrate (cellulose or hemicellulose) is a good candidate of commercial materials [10, 11]. A cellulose fraction naturally forms variable supramolecular structures due to the presence of ordered hydrogen bonds. The structural character in return manifests surface hydrophilicity, biocompatibility, and chirality advantages of cellulose. A limitation of its insolubility is addressed by sulfoethylation or carboxymethylation $[12,13]$. As a result, regioselectively functionalized derivatives are prepared with cellulose [10, 14]. A significant example is the specific oxidation of the fraction by periodates [15]. A dialdehyde product yield allows facile linkage of reactive sulfonates, carboxylic acids, and imines to the cellulose surface [1622]. Another work of cellulose is alkalic and acidic hydration [2], or sulfation by sulfuric acid and sulfate salt. These kinds of cellulose derivates are proposed as catalysts for fine chemical and drug intermediate preparations, and as sorbents for uptake of toxic metal cations.

Stereochemistry influences degrees of cellulose substitution and distribution. Chemical concentration and reaction temperature are key factors of reactions $[16,23$, 24]. In case of the X-ray diffraction measurement, an index of cellulose crystallinity turns out to be critical with respect to chemical reactions [25]. For instance, it is increased with the SVR cellulose fraction while SVR is reacted with hydrochloric acid and oxalic acid [2]. Otherwise, this index 
is declined in a dialdehyde product of cellulose via periodate-oxidation [15, 26]. Sulfation chemistry of cellulose is implicated to substitution process of the D-glucopyranose hydroxyl sites by sulfo groups. It is predicted in a favorable manner because of no disruption of conformational preference of cellulose building blocks. However, details on preparation and characterization of the reaction products are limited and usage of them is not a frequent instance in practice. Even so, cellulose is suggested as a promising material in principle owing to a matter of fact for being adequately abundant in nature, and for providing a good alternative in recommending green architecture through sulfation $[23,26]$.

Here, we focus on two aspects of studies on the SVR cellulose fraction, that is, sulfation chemistry with no preextraction and development of products as heterogeneous catalysts for synthesizing imidazolidine-2,4-dione derivatives. New frontiers such as eco-friendly drilling of waste and green synthesis of heterocyclic compounds are highlighted together with exploitation on regulating surface properties and industrial perspectives in future.

\section{Materials and methods}

\subsection{Preparation of SVR sulfates}

The SVR waste, from Qingxu Jinhua Chuye Co., Ltd, Shanxi in China, is rinsed with water and calcinated anaerobically at $200^{\circ} \mathrm{C}$ It is pulverised to a $74-88 \mu \mathrm{m}$ diameter, sifted, and then collected. The waste at an acidity of $0.082 \mathrm{meq}^{+}$per gram SVR is used. Sulfuric acid, sodium sulfite, sulfamic acid, benzenesulfonic acid, $p$-toluenesulfonic acid, and sodium laurilsulfate are reactive regents. Ferric chloride, aluminum chloride, or zinc chloride is the Lewis acidic salt. All reagents are of AR grade. A feedstock of SVR (50 g), water (15 g), sulfo or sulfoalkyl chemical (0.16-127 mmol), and Lewis acidic salt $(0.5 \mathrm{~g})$ is churned up in dasher machinery. The feedstock is put in a $250 \mathrm{ml}$ porcelain crucible, and sealed with an aluminium foil. After encapsulating the crucible with pink clay layer, it is heated in an atmosphere of $<200^{\circ} \mathrm{C}$ for 3-9 h. Following reaction termination, the feedstock is dispersed in $200 \mathrm{l}$ pure water, filtrated, and washed with 101 water for 3 times. All filtration solution is collected for the assay of sulfation degrees, and solid products are used for characterization and catalysis testing after drying. The rate and degree are calculated as molar and mass changes of the sulfo group during reactions.

\subsection{Assay for SVR sulfation reactions}

If an acid or a salt of the parent acid is the chemical form, total solution collections are titrated directly by sodium hydroxide or hydrochloric acid standards $(0.1 \mathrm{~mol} / \mathrm{l})$. An effect of Lewis acidic salt is deducted from reference control at a same level. Sulfation degrees are calculated by subtracting excess parts of chemicals from total amounts, and obtained as a unit of the meq sulfo per gram SVR. As for sulfamic acid, a ninhydrin-based spectrophotometric assay is performed at $570 \mathrm{~nm}$ using an internal standard method. An acidbase titration method is also used in evaluating acidity changes of sulfates. For example, $1.0 \mathrm{~g}$ SVR sulfate is dispersed in $100 \mathrm{ml}$ sodium hydroxide solution $(0.1 \mathrm{~mol} / \mathrm{l})$ and filtrated. An excess amount of sodium hydroxide is retitrated by hydrochloric acid and the result is calculated.

\subsection{Preparation of imidazolidine-2,4-dione derivatives}

Imidazolidine-2,4-dione, together with its 5,5-diphenyl- and 5-(4-hydroxyphenyl)-substituted derivatives respectively are prepared by synthesizing with urea and $\alpha$-glycolic acid, and the corresponding $\alpha$-glycolic acid substitutes. The $\alpha, \alpha$-dimethyl- $\alpha$-glycolic acid and dimethylolurea are used for preparing 1,3-dimethylol5,5-dimethylimidazolidine-2,4-dione. The SVR sulfate catalysts are dispersed and controlled to a level of 3.0-7.4 mg sulfo in $5 \mathrm{ml}$ solvent mixture ( $\mathrm{N}, \mathrm{N}$-dimethylformamide and water, 1:1 V/V). Typically, $10 \mathrm{mmol}$ urea or dimethylolurea is dissolved in the mixture, and a same amount of $\alpha$-glycolic acid or substitutes is added. Condensation reactions are carried out under agitation for $8 \mathrm{~h}\left(\right.$ at $\left.60^{\circ} \mathrm{C}\right)$, and catalysts are filtrated away from mixtures and washed with water. During process, a vacuum apparatus at a reduced pressure of $0.085 \mathrm{MPa}$ is controlled to prevent materials from oxidation. Sampling is performed at a 0.5-h interval and the samples are assayed with a highperformance liquid chromatography (HPLC) method.

\subsection{Equipments}

Elemental analysis of carbon, hydrogen, nitrogen, and sulfur in SVR is performed using an elemental analyzer (Vario ELcube, Germany). The content of oxygen is analyzed on the 2400 series II oxygen analyzer (Perkin-Elmer Instrument, USA). The FT-IR spectra of SVR sulfates are obtained using a Nicolet Magna 550 IR spectrometer, in the $400-4000 \mathrm{~cm}^{-1}$ range and for each sample, three scans are taken at a resolution of $4 \mathrm{~cm}^{-1}$. Samples are pressed into potassium bromide pellets (1:200). Powder XRD analysis is carried out using a Scintag Model 2000 diffractometer with the $\mathrm{CuK}_{\alpha}$ radiation $(\lambda=1.54056 \AA)$ at $30 \mathrm{kV}$ and $200 \mathrm{~mA}$. The scan increment and $2 \theta$ range are $0.02^{\circ}$ and $10-50^{\circ}$, respectively, using a scan rate at $4.8^{\circ}$ per min. An amount of $100 \mathrm{mg}$ sulfate is filled in a glass slotware and examined. The crystallinity index is obtained by comparing the 002 and amorphous peak intensities [25]. Nitrogen gas physisorption analysis is performed at $-196^{\circ} \mathrm{C}$ using an ASAP 2010 sorption analyzer. Samples are degassed overnight at $150^{\circ} \mathrm{C}$. Specific surface areas are calculated using the BET method at a lower relative pressure of 0.05-0.2. Pore size distribution is obtained by the non-local density functional theory (NLDFT) method assuming cylindrical pore geometry (applying the kernel of metastable NLDFT adsorption isotherm, i.e. adsorption branch) supplied by the Autosorb-1 software 1.55 from Quantachrome Instruments [27]. The HPLC assay in terms of the formed products is performed on the SY-8100 HPLC system equipped with the Venusil MP C18 column (Beifen-Ruili Anal Instrument Co Ltd, Beijing, China). An elution system is the water/acetonitrile/phosphoric acid (90:10:0.01, $\mathrm{V} / \mathrm{V}$ ) mixture. The UV detector is set at $280 \mathrm{~nm}$ and an elution rate 
of $1.0 \mathrm{ml} / \mathrm{min}$ is used. Samples are prepared by centrifuging $400 \mu \mathrm{l}$ reaction medium for $20 \mathrm{~min}$, diluting by 5 -folds, and injecting 10 $\mu \mathrm{l}$ dilutions. An internal standard method is used typically at the concentration of standard panel products (99.9\%, Sigma-Aldrich) of $0.55 \mathrm{mg} / \mathrm{ml}$. An amount of the formed products is determined by quantitative comparison of peak intensities with those for standard panel products in HPLC profiles. After then, a reaction yield and the activity of catalysts are calculated. A repeat performance of the catalyst fitness is evaluated in cycling reactions, and the half-life period (at 50\% residual activity) is also obtained.

\subsection{Statistical analysis}

The statistically significant difference is evaluated using the analysis of variance (ANOVA) and the statistical significance is considered for a p value $<0.05$.

\section{Results and discussion}

\subsection{Chemical composition of SVR}

An elemental composition of carbon, hydrogen, nitrogen, sulfur, and oxygen is $44.22 \pm 0.32,6.10 \pm 0.26,1.68 \pm 0.11$, $0.32 \pm 0.02$, and $39.00 \pm 0.33 \%$, respectively - a comparable result of previous reports [2, 4]. A carbon to nitrogen ratio $(26.32 \pm 2.03)$ in triplicate assays suggests a carbohydrate-rich ingredient of SVR, which is composed naturally of cellulose, hemicellulose, and lignin [2]. The polymeric ingredient makes up the primary and secondary structures of cell walls, and forms an embedded network of cellulose fibril with hemicellulose and lignin. The crosslinkage network gets to elimination of water away from cell walls and formation of a hydrophobic surface that limits accessibility of reactive chemicals. The hydrophobic surface plays a major contribution to characteristics of secondary structures of cell walls.

\subsection{SVR sulfation kinetics}

Sulfating SVR in solvent systems is difficult because of the dynamic unfavourablity of the reactions. Another disadvantage is generated by an environmentally nonfriendly effect of large solvent volumes. We design here a sealed wet curing technique for attempting synthesis of sulfates, under controllable conditions for chemical quantities almost equalling to stoichiometric ratios and small amounts of solvent. In Figure 1, a rate is only $20.12 \mu \mathrm{mol}$ $(\mathrm{g} \times \mathrm{h})^{-1}$ (control) of a SVR reaction with sulfuric acid at $180^{\circ} \mathrm{C}$. It is sped up by $2.32-2.63$ folds while in the presence of Lewis acidic salt (i.e. sulfation rates of 52.92, 48.89,

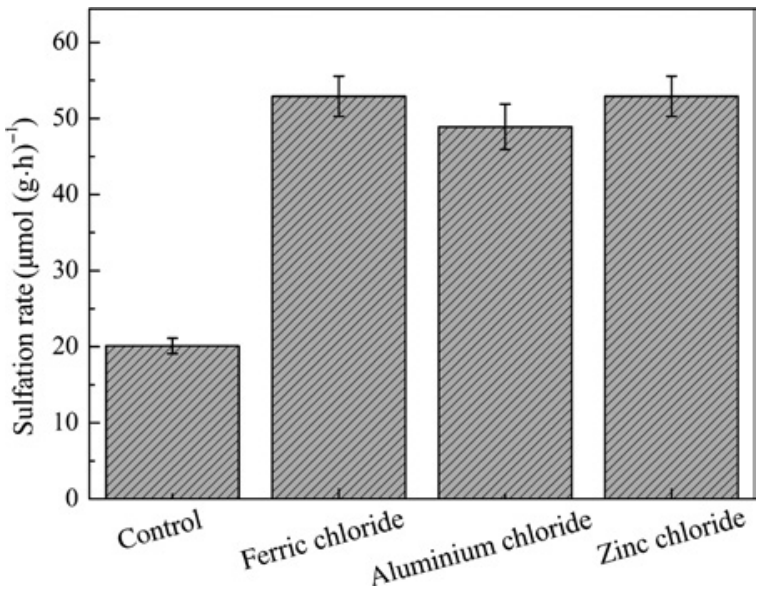

Figure 1: Effects of the sulfation reaction kinetics of Sorghum vinegar residue (SVR) and sulfuric acid, initiated by Lewis acidic salts for ferric chloride, aluminum chloride and zinc chloride, respectively. A feedstock ratio of $1.0 \mathrm{mmol} / \mathrm{g} \mathrm{SVR}$, and a radical initiator amount of $1.0 \%$ were used. The reaction was carried out under wet curing at $180^{\circ} \mathrm{C}$.

and $52.92 \mu \mathrm{mol}(\mathrm{g} \times \mathrm{h})^{-1}$ for anhydrous ferric chloride, aluminium chloride, and zinc chloride, respectively). The sulfation process is predicted to follow a Fischer esterification mechanism $[23,26]$, by which the hydrogen atom at D-glucopyranose hydroxymethyl sites is substituted by dehydroxylated sulfuric acid. The cellulose fraction and sulfuric acid are reactive donors for producing the hydrogen group and the hydroxyl free radical. Lewis acidic salt is used as a typical initiator for sulfation chemistry, probably owing to its fairly strong acidity [28]. Because Figure 1 shows the sulfation rate of anhydrous ferric chloride and zinc chloride as being extremes (whereas practically a same level), we enroll anhydrous ferric chloride as an example for accelerating SVR reaction kinetics.

The concentration-dependent kinetics of SVR sulfation is evaluated with sulfuric acid and sodium sulfite chemicals (Figure 2). The saturation kinetics reached at a quantity of chemicals above $1.0 \mathrm{mmol}$ sulfo per gram SVR. Under free radical initiation, the reaction rate begins asymptotic variation to a steady state of 53.1 and $48.0 \mu \mathrm{mol}(\mathrm{g} \times \mathrm{h})^{-1}$ and a change above the stoichiometric equivalence point does not lead to an increase of the rate anymore [29]. An evaluation of saturation kinetics of the SVR reaction is good guidance for achieving the optimum sulfation degree of SVR, and at the same time it allows the low pollution potential from an excess amount of chemicals above the kinetics.

An effect of the reaction temperature is seen in Figure 3. Each reaction achieves a favorable temperature point for the maximal degree. A low point $\left(70^{\circ} \mathrm{C}\right.$ or $\left.90^{\circ} \mathrm{C}\right)$ of SVR sulfation with sodium sulfite and sulfamic acid 


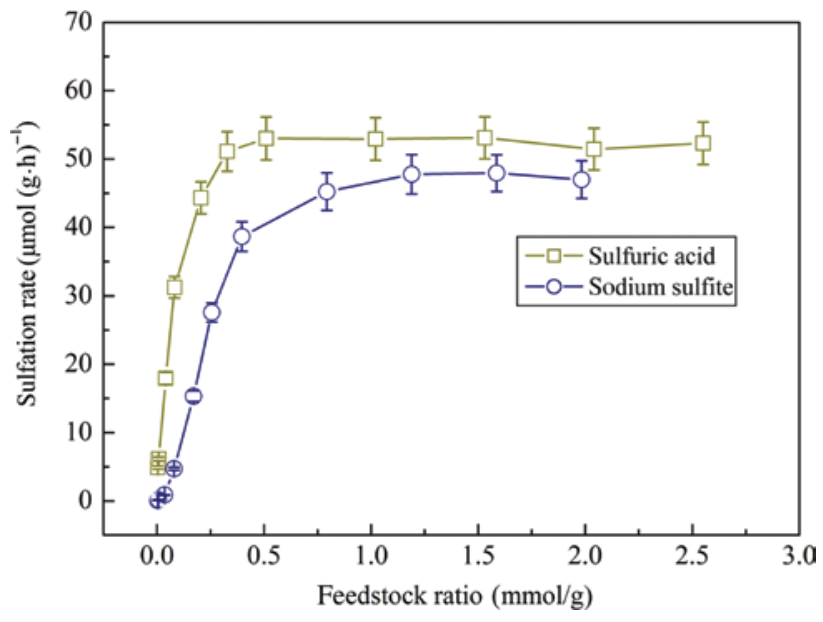

Figure 2: Sulfation reaction kinetics of SVR with sulfuric acid and sodium sulfite, respectively, at different feedstock ratios. The amount of anhydrous ferric chloride used was $1.0 \%$, and temperatures for wet curing were $180^{\circ} \mathrm{C}$ and $70^{\circ} \mathrm{C}$.

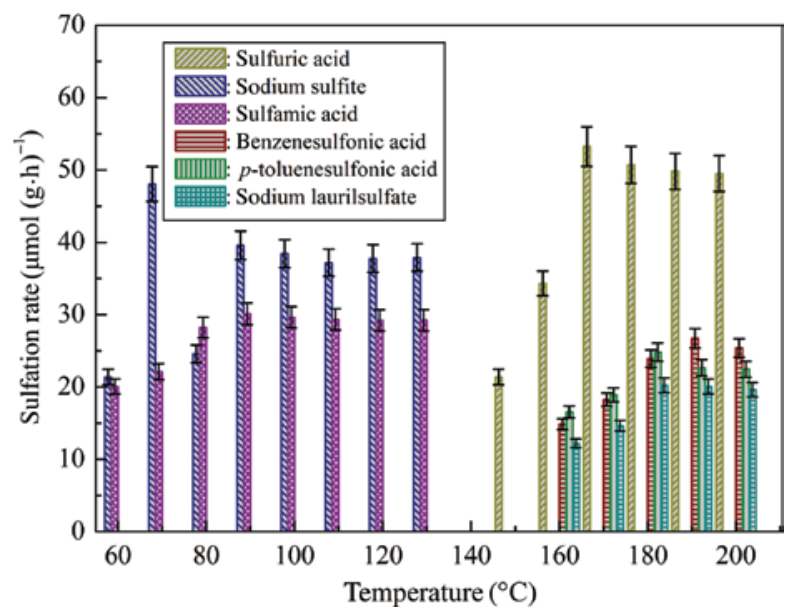

Figure 3: Sulfation rates of SVR with six sulfo and sulfoalkyl chemicals at different temperatures. Initial reaction rates were obtained from the sulfate production amounts at a wet curing period of about $1.0 \mathrm{~h}$.

suggests fast production of hydrogen sulfite free radical or good efficiency for preventing sulfamic acid from decomposition [30]. Organic sulfoalkyl chemicals yield slow reaction kinetics in a rank of sulfuric acid $>$ sodium sulfite $>$ sulfamic acid $>$ benzenesulfonic acid $>p$-toluenesulfonic acid $>$ sodium laurilsulfate. The maximal reaction degrees are listed in Table 1. An interesting structural network of SVR is constructed by means of the chemical linkage of sulfo and sulfoalkyl groups. In a word, green synthesis of sulfates has been achieved through curing feedstock in a sealed condition. Wet curing rather than typical solvent heat transfer is also a rational drilling technique on exploiting other carbohydrate-rich wastes and getting more novel derivatives.

\subsection{Characterization of SVR sulfates}

The FT-IR spectra of SVR and sulfates are compared in Figure 4. The spectra of sulfates (curves $b-g$ ) illustrate derivatization to the spectrum of SVR (curve $a$ ), and some differences are found. New signals (738.7, 837.5, and $\left.1267.2 \mathrm{~cm}^{-1}\right)$ and division of a peak $\left(1068.4 \mathrm{~cm}^{-1}\right)$ into doublet $\left(1040.2\right.$ and $\left.1157.6 \mathrm{~cm}^{-1}\right)$ appeared in the spectra of sulfates. A band at $1040.2 \mathrm{~cm}^{-1}$ is attributed to a response of symmetric stretching vibration of the $\mathrm{O}=\mathrm{S}=\mathrm{O}$ group, while that at $1267.2 \mathrm{~cm}^{-1}$ is due to asymmetric stretching vibration of $\mathrm{O}=\mathrm{S}=\mathrm{O}$ [31]. The band at $738.7 \mathrm{~cm}^{-1}$ is derived from stretching vibration of the $\mathrm{S}-\mathrm{C}$ group and a small one around $837.5 \mathrm{~cm}^{-1}$ is ascribed to deformation vibration of $\mathrm{C}=\mathrm{H}$ and $\mathrm{CH}_{2}$ in benzenesulfonic acid (curve $e$ ), $p$-toluenesulfonic acid (curve $f$ ), and laurilsulfate (curve $g$ ) [32]. The SVR sulfation formation with sulfamic acid (curve $d$ ) also shows an absorption signal at about $837.5 \mathrm{~cm}^{-1}$, but that with sulfuric acid (curve $b$ ) or sodium sulfite (curve $c$ ) does not show. It is interesting to describe a new peak $\left(1157.6 \mathrm{~cm}^{-1}\right)$ in the spectra of sulfates. A relatively strong intensity is the resultant of the absorption of the $\mathrm{C}-\mathrm{O}$ stretching vibration, which is an evidence for the introduction of the $\mathrm{C}-\mathrm{O}$ group [32]. Compared to the spectrum of SVR, changes in both the peak position and the intensity suggest $\mathrm{C}-\mathrm{O}-\mathrm{S}$ linkage formation and also reflect a sulfation pathway. Besides, a broad band at $3410 \mathrm{~cm}^{-1}$ is a nestification of the $\mathrm{OH}, \mathrm{H}$ and $\mathrm{NH}$ stretching vibrations. The dual peaks around $3000 \mathrm{~cm}^{-1}$ are due to $\mathrm{CH}$ and $\mathrm{CH}_{2}$ vibrations [33].

Appearance of a band centered at $738.7 \mathrm{~cm}^{-1}$ is the most reliable indication of the $\mathrm{S}-\mathrm{C}$ bond formation mostly introduced in sulfonation process [31-33], or seldom in the process of sulfation with sulfoalkyl chemicals. Cellulose sulfonation is believed to happen in the chemical process of acute oxidation [15], during which the D-glucopyranose backbone is disrupted following new S-C bond formation. In the case of a sealed condition, sulfation process is proposed rather than sulfonation because no oxidative reagent is added. The proposition is suggested by appearance of the $738.7 \mathrm{~cm}^{-1}$ peak only in spectra of some samples but not all. For example, the band is not showed in the spectra of SVR sulfates formed with sulfuric acid, sodium sulfite, and sulfamic acid. In consideration of these discussions, a structural diagram of sulfates shall be suggested as what is shown in Scheme 1. However, highlighting the cellulose fraction reaction shall not be 


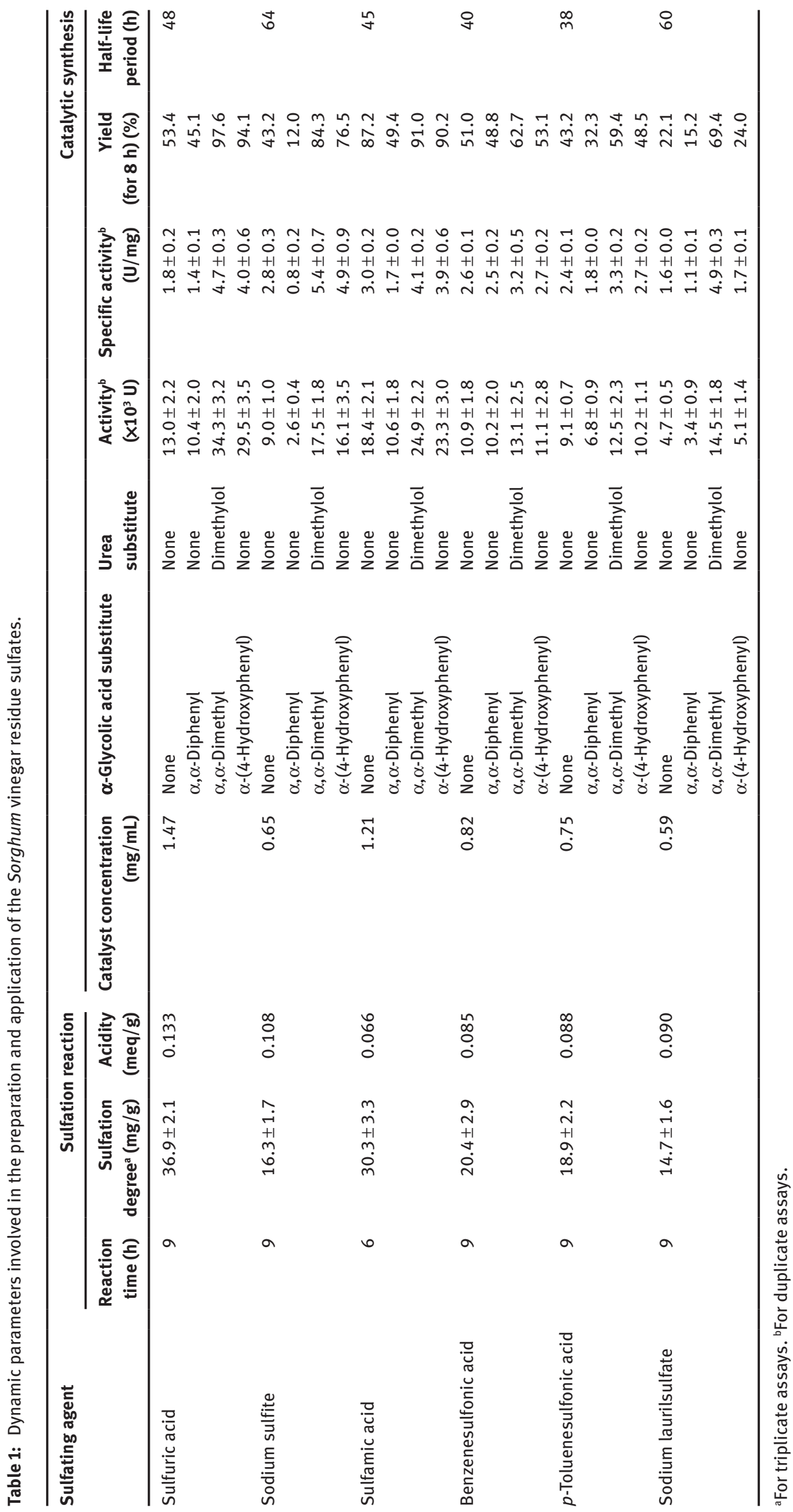




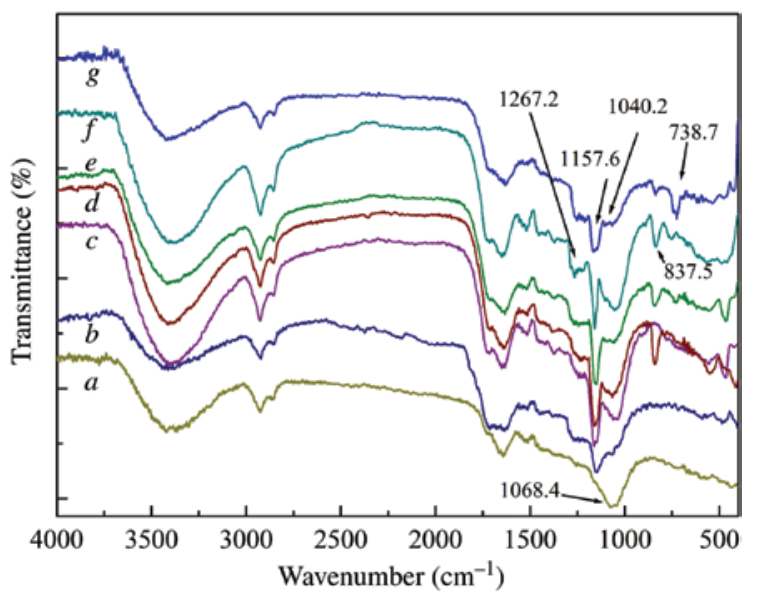

Figure 4: The FT-IR spectra of native SVR (curve $a$ ), and the sulfates formed with sulfuric acid (curve $b$ ), sodium sulfite (curve $c$ ), sulfamic acid (curve $d$ ), benzenesulfonic acid (curve $e$ ), $p$-toluenesulfonic acid (curve $f$ ) and sodium laurylsulfate (curve $g$ ), respectively. Samples were made in potassium bromide thin pellets (1:200), and obtained as an average for three scans.

whole scenario because of random substitution of sulfo and sulfoalkyl groups, and average probability of SVR distribution.

Powder XRD curves of SVR (curve $a$ ) and sulfates (curves $b-g$ ) are shown in Figure 5. In spite of a low signal-to-noise ratio, a crystallinity index of SVR is estimated roughly at $28.18 \%$ by peak fit and evaluation of relative intensities of the 002 and amorphous signals $\left(2 \theta=21.3^{\circ}\right.$ and $\left.18.7^{\circ}\right)$ [25]. Relative changes are expressed as a percentage unit for subtracting two signal intensities and dividing the 002 signal intensity. Although variation depending on a choice of estimation methods, the index obtained by this way in XRD curves is used widely to describe a relative amount of crystalline material in cellulose chains via the two-phase model for ordered crystalline and less ordered amorphous regions. Furthermore, it is used to interpret changes of the cellulose structure after treatments $[2,8,15,25,27]$. It is seen in Figure 5, due to more broad and flat patterns, crystallinity indices of sulfates are decreased to extremes of $4.90 \%$ (with sodium laurilsulfate) and $12.89 \%$ (with sodium sulfite), respectively. These changes, namely a reduction of crystallinity in the sulfated SVR structure, support reaction preference of chemicals with amorphous texture of the cellulose fraction, because it has small toughness [34]. Alternatively, an ordered cross-linked structure of crystalline texture is disrupted at a heating condition [25].

For a possible purpose of catalysis, the adsorptiondesorption isotherm and pore size distribution of SVR are compared with the sulfate by sulfuric acid (Figure 6A, B). Typically, a type IV classification of physical adsorption isotherm indicates a mesoporous property and a sharp step to capillary condensation [35]. Hysteresis loop in a high relative pressure range (0.6-1.0) suggests strong fluidwall forces. Brunauer-Emmett-Teller (BET) specific surface areas are 14.55 and $16.72 \mathrm{~m}^{2} / \mathrm{g}$ for two materials. A change in the specific surface area is a resultant of the surface reaction of amorphous cellulose, which is a consistent variation to XRD patterns. The soft textural structure contributes to a high BET area of the noncrystalline cellulose fraction. In addition, chemical linkage of $\mathrm{C}-\mathrm{O}-\mathrm{S}$ leads to an increased BET area of the fraction when it is determined in a relative pressure range beyond hysteresis loop. This effect is reflected by distribution of an average pore size

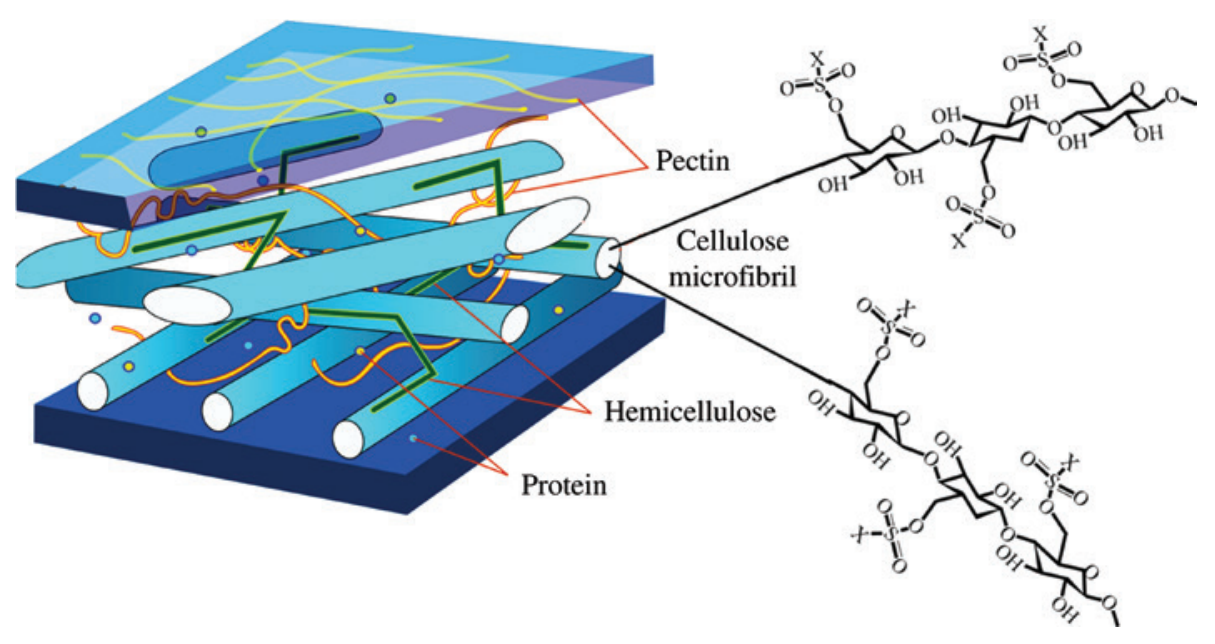

Scheme 1: A proposed diagram for the structures of Sorghum vinegar residue (SVR) sulfates developed in the work, highlighting substitution of the sulfo or sulfoalkyl chemicals with the cellulose fraction in SVR. $X=$ hydroxyl, none, amido, phenyl, 4-methylphenyl, and lauril groups, respectively. 


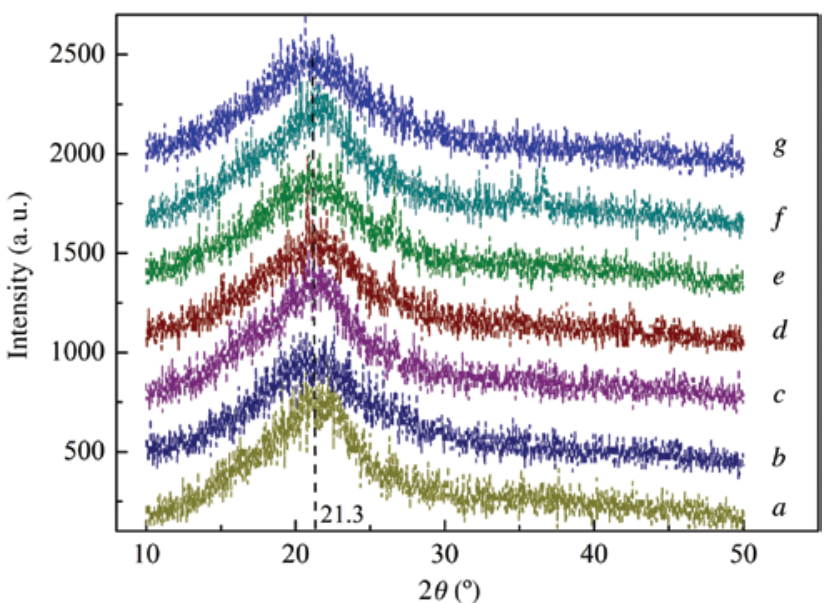

Figure 5: The XRD patterns of the SVR without treatment (curve $a$ ), and with treatment by sulfuric acid (curve $b$ ), sodium sulfite (curve $c$ ), sulfamic acid (curve $d$ ), benzenesulfonic acid (curve $e$ ), $p$-toluenesulfonic acid (curve $f$ ), and sodium laurylsulfate (curve $g$ ), respectively.

calculated from the volume adsorbed at a 0.95 relative pressure. As seen in Figure 6 insets, a single contiguous pore space is 310.1 and $306.7 \mathrm{~nm}$ for two materials. In comparison with a result of SVR, dehydroxylated sulfuric acid is proposed to fill in an inner layer of sulfate pores. It is important to note that the isotherm of sulfate shows particularly well-developed mesoporosity, which is expressed by a sharp capillary condensation step and hysteresis loop, and characteristic for ordered cylindrical-like pores. This result makes the material a possible catalyst in practice.

\subsection{Catalysis tests}

Catalysis tests of sulfates are evaluated in reaction systems for preparing diverse imidazolidine-2,4-dione derivatives. Different engagement of substance substitutes shall facilitate screening of catalysts and help to realize theoretical elucidation. Derivatives are found widely in medical usages like anticonvulsant, antiepileptic and antiseizure drug, as well as antimicrobial formaldehyde releaser and others [36]. Strong acid, phosphoric anhydride, potassium hydroxide, and heteropolyoxometalate are attempted as catalysts in laboratory [36]. However, they have disadvantages of slow reaction kinetics, severe conditions, and side effects. Other limitations are hard recyclability of catalysts, easy corrodibility on reactors, and high pollution towards environment. A new catalyst system for green synthesis with easy work-up is an important challenge. A molecular mechanism on synthesizing imidazolidine2,4-dione derivatives is depicted (Scheme 2).
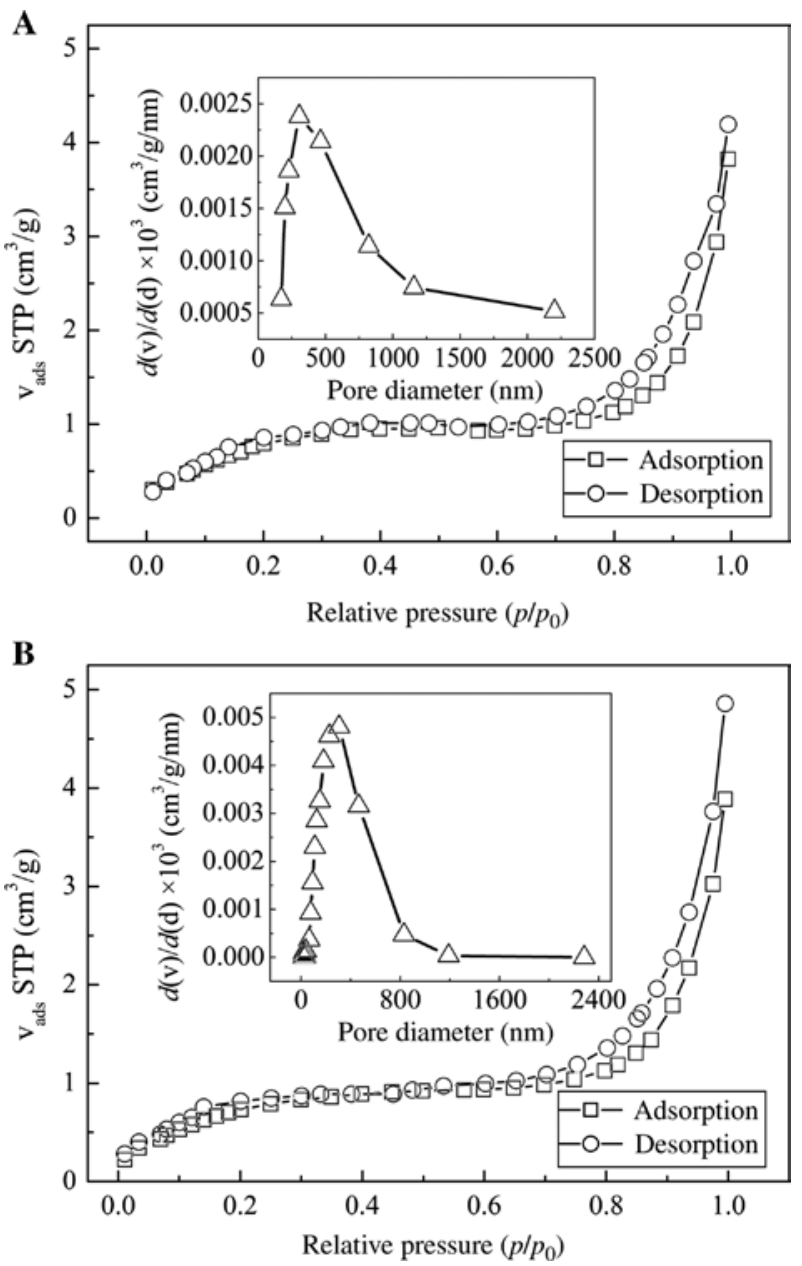

Figure 6: Nitrogen gas physisorption isotherms $\left(-196^{\circ} \mathrm{C}\right)$ and the corresponding pore size distributions (insets) of (A) native SVR and (B) sulfate material synthesized with sulfuric acid. Pore size distributions were calculated from the adsorption branch of the isotherm using the NLDFT method.

The SVR sulfates are obtained in solid states; dispersion in liquid media indicates a heterogeneous action of them. An amount of imidazolidine-2,4-dione derivatives is determined by the HPLC assay, and elution patterns of individual standards are shown in Figure 7. The retention time is correlated to hydrophobic affinity alternatives of derivatives with the octadecyl carbon chain (C18)-bonded silica substance in the HPLC column. The hydrophobic derivative achieves a slow elution.

Using an internal standard method, elution peak intensities are ascribed as control for quantitative determinations. Dynamic parameters of reactions are listed in Table 1. Catalytic and specific activities of sulfates are recovered at 2.6-34.3 $\left(\times 10^{3} \mathrm{U}\right)$ and $0.8-5.4 \mathrm{U} / \mathrm{mg}$, when the level of sulfates is $0.6-1.48 \mathrm{mg} / \mathrm{l}$. The minimal and maximal extremes of catalytic activities are observed 


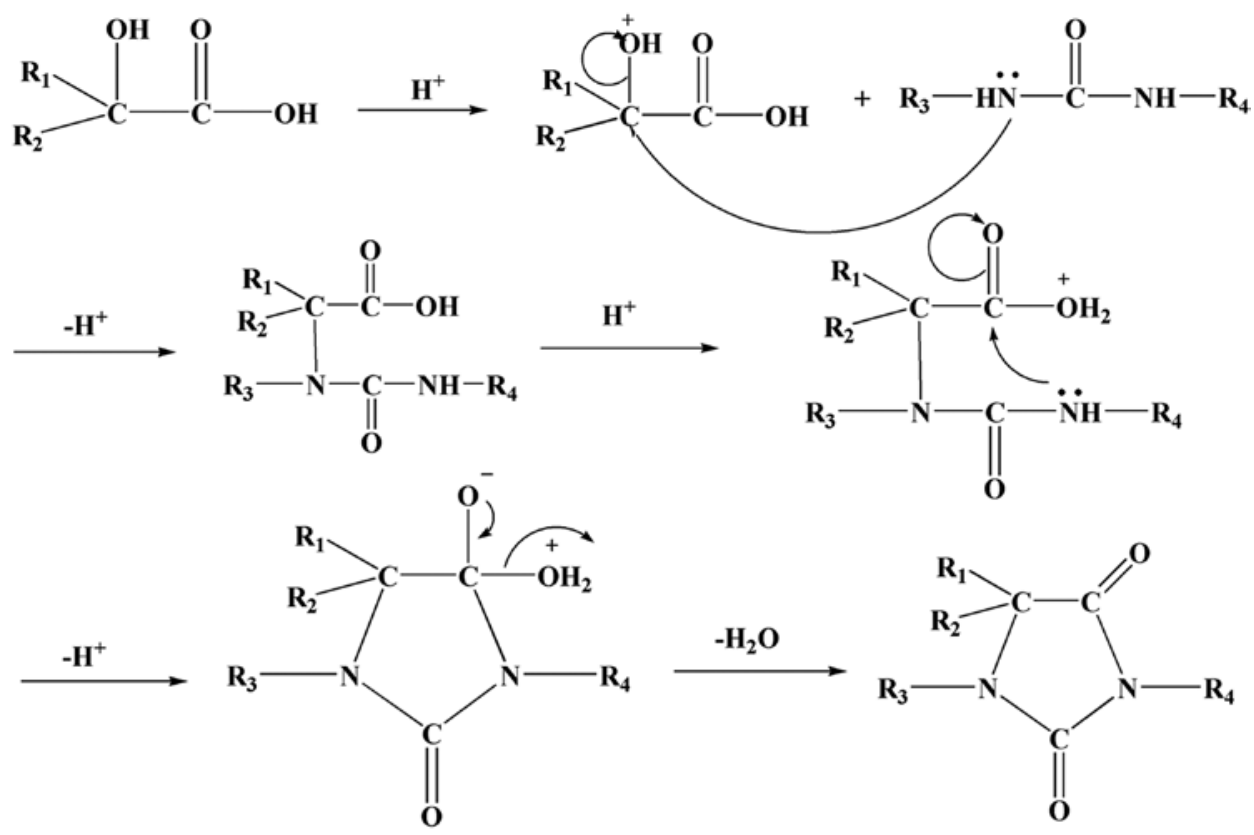

\section{$\mathbf{R}_{1}=$ hydrogen, methyl, phenyl, and phenolic groups; $\mathbf{R}_{2}=$ hydrogen, methyl and phenyl groups; $R_{3}, R_{4}=$ hydrogen and methoxyl group.}

Scheme 2: A molecular mechanistic diagram of the solid acid-mediated catalysis in the preparation of imidazolidine-2,4-dione derivatives.

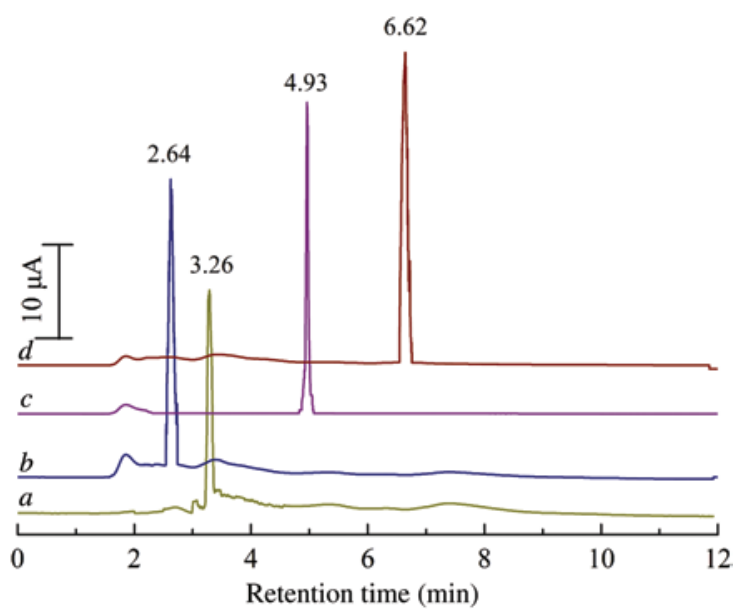

Figure 7: The HPLC profiles of imidazolidine-2,4-dione (curve a), 1,3-dimethylol-5,5-dimethylimidazolidine-2,4-dione (curve b), 5-(4-hydroxyphenyl)imidazolidine-2,4-dione (curve $c$ ), and 5,5-diphenylimidazolidine-2,4-dione (curve $d$ ) standards at a concentration of $0.55 \mathrm{mg} / \mathrm{ml}$ in a water/acetonitrile/phosphoric acid (90:10:0.01, V/V) mixture system. UV-Vis detector was set at $280 \mathrm{~nm}$; the injection volume and the elution rate were $10 \mu \mathrm{l}$ and $1.0 \mathrm{ml} / \mathrm{min}$.

with catalysts formed by sodium sulfite and sulfuric acid, during the process of 5,5-diphenylimidazolidine-2,4-dione and 1,3-dimethylol-5,5-dimethylimidazolidine-2,4-dione preparation. But, extreme limitations of specific activities are found for the catalyst formed by sodium sulfite in the case of two preparations. Variation on catalytic and specific activities is not consistent due to an emendation effect of the catalyst concentration.

Chemical catalysis is brought into a fairly complex process. We try to perform basic exploitation with respect to catalyst and substrate aspects. At first, hydrophobicity of substrates is correlated to the property of reaction products. Due to a high degree (Figure 7), preparing 5,5-diphenylimidazolidine-2,4-dione favors a hydrophobic catalyst. The specific activity of sulfates by different chemicals is changed by benzenesulfonic acid $>p$-toluenesulfonic acid $>$ sulfamic acid $>$ sulfuric acid $>$ sodium laurilsulfate $>$ sodium sulfite. The sulfate by a laurilsulfate group shows a relatively predominant activity probably because of an anionic or emulsifiable group [37]. A reverse order of specific activities is observed for preparing the most hydrophilic 1,3-dimethylol-5,5-dimethylimidazolidine2,4-dione, with the minimal extreme of catalysts formed by benzenesulfonic and $p$-toluenesulfonic groups. Besides, acidity is a critical parameter of substrates. In Scheme 2, activating $\alpha$-glycolic acid substitutes to protonation states is necessary during initial chemical process [36]. As pKa values of $\alpha$-glycolic acid, $\alpha, \alpha$-diphenyl- $\alpha$-glycolic acid, $\alpha, \alpha$-dimethyl- $\alpha$-glycolic acid, and $\alpha$-(4-hydroxyphenyl)$\alpha$-glycolic acid are 3.6, 3.05, 4.72, and 4.50 [38], protonation dynamics is assumed to delimit catalysis. An acidity degree of $0.066-0.133$ meq per gram sulfate (Table 1) 
reveals a proton donor capacity of catalysts. The capacity facilitates protonation activation of substitutes by sulfates, which is a like action of general macroreticulartype cation exchange resins (with strongly acidic sulfonic group) mostly in self-condensation instances. The specific activity of catalysts shall be decreased by an increase in the order of acidity of substitutes, which is the resultant of easier protonation by a weak acid than a strong one [37]. But, even if a same level of SVR is controlled, an acidity effect of sulfates is hard to be distinguished due to the synergistic action with hydrophobicity [39]. A preliminary study indicates that native SVR gives about 3.5-8.3\% specific activity of sulfate formed by sulfuric acid, when catalyzing the imidazolidine-2,4-dione preparation. However, this result shall not be considered as a sole effect of acidity because of the average distribution of $\mathrm{H}^{+}$sites [10, 12].

Under the most efficient catalysis, individual reactions achieve maximal yields ( $8 \mathrm{~h}$ ) of 49.4\%, 87.2\%, 94.1\%, and $97.6 \%$ for four imidazolidine-2,4-dione derivatives (Table 1). A good catalysis action is realized with these sulfates. The work-up of recovering catalysts to be reused is simply achieved through filtration. As a result, reproducibility is guaranteed in new reaction batches and catalysts are separated and cycled repeatedly. The half-life period of catalyst fitness is 38-64 h. Variation on fitness may be ascribed as the resultant for different inactivation towards reaction medium interactions or swelling capacity-mediated degradation in solvents $[2,16]$. A limitation of relatively short half-life is addressed repeatedly by curing activation of catalysts. Residual specific activities are retained $88 \%, 73 \%$, and $62 \%$ of initiation during the first to third activation repeats of sulfate formed by sulfuric acid. Other factors of catalysts such as the BET area, pore space, and surface stereochemistry will be studied in future.

\section{Conclusions}

In this work, a new interesting structural network of SVR sulfates with inorganic/organic sulfo and sulfoalkyl chemicals has been constructed for the exploitation of waste reutilization together with catalytic application. Regulatory surfaces achieve good catalysis of sulfates towards synthesis of imidazolidine-2,4-dione derivatives. A matching network of catalyst favorability ratings was addressed by engagement of different reactant substitutes. Information on this work paves a green routine for reusing agroindustrial wastes but not limiting to this natural material in an industrial processing field, by selecting sulfated wastes in the network, or alternatively, by developing new ones that are suitable for application in a better way.

Acknowledgements: This work was supported by the Shanxi Special Foundation for Alteration of Large-Scale Scientific Instruments (project no.: 20150524-13), and the Shanxi Natural Science Foundation (project no.: 2012011002-3).

Conflict of interest statement: The authors have declared no conflicts of interest.

\section{References}

[1] Nakayama T. J. Biochem. 1959, 46, 1217-1225.

[2] Wang ZB, Shao SP, Zhang CS, Lu DL, Ma HL, Ren X. Int. J. Hydrogen Energ. 2015, 40, 4494-4501.

[3] Li L, Feng L, Zhang R, He Y, Wang W, Chen C, Liu G. Bioresour. Technol. 2015, 186, 338-342.

[4] Feng L, Li Y, Chen C, Liu X, Xiao X, Ma X, Zhang R, He Y, Liu G. BioResources 2013, 8, 2487-2498.

[5] Chen L, Yang X, Raza W, Luo J, Zhang F, Shen Q. Bioresour. Technol. 2011, 102, 3900-3910.

[6] Wang ZH, Dong XF, Zhang GQ, Tong JM, Zhang Q, Xu SZ. Waste Manag. Res. 2011, 29, 1262-1270.

[7] Liu J, Yang JC. For. Stud. China 2006, 8, 22-25.

[8] Algar I, Fernandes SCM, Mondragon G, Castro C, Garcin-Astrain C, Gabilondo N, Retegi A, Eceiza A. J. Appl. Polym. Sci. 2015, 132, 41237-41245.

[9] Li K, Chen H, Yu H, Zhu H, Mao Q, Ma X, Zhao Z, Xiao T. BioResources 2014, 9, 4993-5006.

[10] Klemm D, Heinze T, Philipp B, Wagenknecht W. Acta Polym. 1997, 48, 277-297.

[11] Ragauskas AJ, Williams CK, Davison BH, Britovsek G, Cairney J, Eckert CA Jr., Frederick WJ, Hallett JP, Leak DJ, Liotta CL, Mielenz JR, Murphy R, Templer R, Tschaplinski T. Science 2006, 311, 484-489.

[12] Kulicke W-M, Kull AH, Kull W, Thielking H, Engelhardt J, Pannek JB. Polymer 1996, 37, 2723-2731.

[13] Gelman RA. J. Appl. Polym. Sci. 1982, 27, 2957-2964.

[14] Koschella A, Fenn D, Illy N, Heinze T. Macromol. Symp. 2006, 244, 59-73.

[15] Xiong J, Ye J, He X, Wu Z. Polym. Mater. Sci. Eng. 2000, 3, 172-175.

[16] Rajalaxmi D, Jiang N, Leslie G, Ragauskas AJ. Carbohydr. Res. 2010, 345, 284-290.

[17] Saito T, Kimura S, Nishiyama Y, Isogai A. Biomacromolecules 2007, 8, 2485-2491.

[18] Habibi Y, Vignon MR. Cellulose 2008, 15, 177-185.

[19] Maekawa E, Koshijima T. J. Appl. Polym. Sci. 1990, 40, 1601-1613.

[20] Casu B, Naggi A, Torri G, Allegra G, Meille SV, Cosani A, Terbojevich M. Macromolecules 1985, 18, 2762-2767.

[21] Koshijima T, Tanaka R, Muraki E, Yamada A, Yaku F. Cellulose Chem. Technol. 1973, 7, 197-208.

[22] Maekawa E, Koshijima T. J. Appl. Polym. Sci. 1991, 42, 169-178. 
[23] Kim UJ, Kuga S, Wada M, Okano T, Kondo T. Biomacromolecules 2000, 1, 488-492.

[24] Varma AJ, Chavan VB. Polym. Degrad. Stability 1995, 49, 245-250.

[25] Park S, Baker JO, Himmel ME, Parilla PA, Johnson DK. Biotechnol. Biofuels 2010, 3, 10.

[26] Klemm D, Heublein B, Fink HP, Bohn A. Angew. Chem. Int. Ed. Engl. 2005, 44, 3358-3393.

[27] Landers J, Gor GYu, Neimark AV. Colloids Surf. A 2013, 437, 3-32.

[28] Carlini C, Giuttari M, Galletti AMR, Sbrana G, Armaroli T, Busca G. Appl. Catal. A-Gen. 1999, 183, 295-302.

[29] Platt JR. Science 1964, 146, 347-353.

[30] Benson GA, Spillane WJ. Chem. Rev. 1980, 80, 151-186.

[31] Cabassi F, Casu B, Perlin AS. Carbohydr. Res. 1979, 63, 1-11.

[32] Socrates G, Eds. Infrared and Raman Characteristic Group Frequencies, 3rd ed., Wiley: England, 2001.

[33] Zhang K, Brendler E, Gebauer K, Gruner M, Fischer S. Carbohydr. Polym. 2011, 83, 616-622.

[34] Sannigrahi P, Miller SJ, Ragauskas AJ. Carbohydr. Res. 2010, 345, 965-970.

[35] Balbuena PB, Gubbins KE. Langmuir 1993, 9, 1801-1814.

[36] Micheletti G, Delpivo C, Baccolini G. Green Chem. Lett. Rev. 2013, 6, 135-139.

[37] Bondi CAM, Marks JL, Wroblewski LB, Raatikainen HS, Lenox SR, Gebhardt KE. Environ. Health Insights 2015, 9, 27-32.

[38] Perrin DD, Dempsey B, Serjeant EP. In $p K_{\alpha}$ Prediction for Organic Acids and Bases, 2nd ed., Perrin, DD, Ed., Chapman \& Hall: London, 1981, Vol. 3, p. 21.

[39] Venu Madhav J, Thirupathi Reddy Y, Narsimha Reddy P, Nikhil Reddy M, Kuarm S, Crooks PA, Rajitha B. J. Mol. Catal. A Chem. 2009, 304, 85-87.

\section{Bionotes}

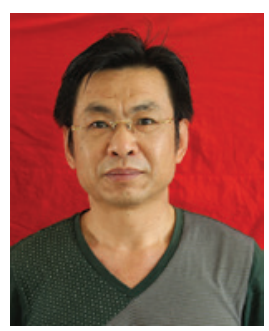

Yuanbiao Qiao

Yuanbiao Qiao is an academic leader at the Graduate Institute of Pharmaceutical Chemistry, Luliang University, Shanxi, P.R. China. His current research areas cover organic synthesis, asymmetry catalysis, and natural product chemistry.

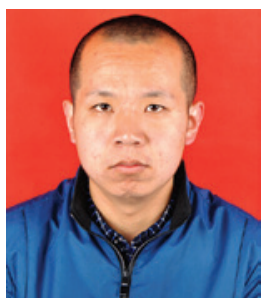

Wenhao Qin

Wenhao Qin has a Bachelor's degree and works in the Department of Chemistry and Chemical Engineering, Luliang University, Shanxi, P.R. China. His current research area is carbohydrate chemistry.

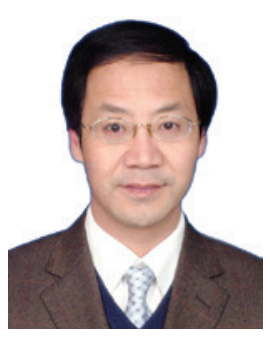

Qingshan Li

Qingshan Li is a pharmacological specialist in the College of Traditional Chinese Pharmacology, Shanxi University of Traditional Chinese Medicine, Shanxi, P.R. China. His research areas are infectious disease chemotherapy, drug discovery and development, and bioinformatics. 